\title{
Protein-Protein Interaction Network Analysis and Identification of Key Players in nor-NOHA and NOHA Mediated Pathways for Treatment of Cancer through Arginase Inhibition: Insights from Systems Biology
}

\author{
Ishtiaque Ahammad ${ }^{1 *}$ \\ 1. Department of Biochemistry and Microbiology, North South University, Dhaka, \\ Bangladesh. \\ *Corresponding author. Email: ishtiaque.ahammad@ northsouth.edu
}

\begin{abstract}
L-arginine is involved in a number of biological processes in our bodies. Metabolism of Larginine by the enzyme arginase has been found to be associated with cancer cell proliferation. Arginase inhibition has been proposed as a potential therapeutic means to inhibit this process. Nhydroxy-nor-L-Arg (nor-NOHA) and N (omega)-hydroxy-L-arginine (NOHA) has shown promise in inhibiting cancer progression through arginase inhibition. In this study, nor-NOHA and NOHA-associated genes and proteins were analyzed with several Bioinformatics and Systems Biology tools to identify the associated pathways and the key players involved so that a more comprehensive view of the molecular mechanisms including the regulatory mechanisms can be achieved and more potential targets for treatment of cancer can be discovered. Based on the analyses carried out, 3 significant modules have been identified from the PPI network. Five pathways/processes have been found to be significantly associated with nor-NOHA and NOHA associated genes. Out of the 1996 proteins in the PPI network, 4 have been identified as hub proteins-SOD, SOD1, AMD1, and NOS2. These 4 proteins have been implicated in cancer by other studies. Thus, this study provided further validation into the claim of these 4 proteins being potential targets for cancer treatment.
\end{abstract}

\section{Keywords}

Arginase; nor-NOHA; NOHA; Cancer

\section{Introduction}

L-arginine, a basic amino acid, has a major role in a number of systems in our bodies including the immune system, particularly on the proliferation of T lymphocytes [1]. The enzyme arginase metabolizes L-arginine to L-ornithine which is important in the biochemical pathways involved in cell proliferation [2]. Two isoforms of arginase exists: arginase I, and arginase II. Arginase I is 
a cytosolic enzyme which is primarily found in hepatocytes, erythrocytes, and granulocytes [3], [4] and Arginase II is found in the mitochondria of various tissues, including kidney, brain, and prostate [5], [6]. Arginase II activity has been shown to be increased in breast, colon, and prostate cancer [7], [8]

Arginase inhibition has been found to suppress proliferation of breast cancer cells. Therefore it is being considered as a new therapeutic target for suppressing breast cancer cell growth. In tumor cells with elevated arginase activity, arginase inhibitors can be a potential therapeutic option for treating cancer [9].

$\mathrm{N}$ (omega)-hydroxy-L-arginine (NOHA) has been found to selectively inhibit cell proliferation and induce apoptosis in high arginase expressing MDA-MB-468 cells [7]. NOHA is also involved in the modulation of $\mathrm{T}$ cell receptor $\mathrm{CD} 3$ zeta which undergoes down-regulation in cancer [7].

Injection of N-hydroxy-nor-L-Arg (nor-NOHA) blocked growth of lung cancer cells in mice [10]. In another study, nor-NOHA significantly $(\mathrm{P}=0.01)$ reduced arginase II activity and suppressed growth of cells with high arginase activity in renal carcinoma [9]

The purpose of this study was to identify crucial genes and proteins involved in the nor-NOHA and NOHA mediated pathways in order to shed more light on their mechanism of action and the key players involved. For this, nor-NOHA and NOHA-associated genes from several databases was identified. And multiple Bioinformatics and Systems Biology tools have been used to identify more nor-NOHA and NOHA-associated key genes. They included pathway enrichment analysis, protein-protein interaction (PPI) network, module analysis and transcriptional regulatory network analysis.

\section{Methods}

\section{Nor-NOHA and NOHA-Associated Genes}

"nor-NOHA" and "NOHA" were used as keywords to search in three databases for genes associated with these compounds and the results were combined to build a set of Nor-NOHA and NOHA-associated genes. The three databases used were-

- GeneCards (version 3.0), a searchable, integrative database which provides comprehensive information on all annotated and predicted human genes [11].

- Search Tool for Interacting Chemicals (STICH, version 5.0), a database of known and predicted interactions between chemicals and proteins [12]

- Comparative Toxicogenomics Database (CTD), which provides manually curated information concerning chemical-gene/protein interactions, chemical-disease as well as gene-disease relationships was used [13]. 


\section{PPI Network}

Two databases were combined to predict the Protein-Protein Interaction (PPI) pairs among the nor-NOHA and NOHA-associated genes. The databases used were-

- Biological General Repository for Interaction Datasets (BioGRID, version 3.4, https://wiki.thebiogrid.org/) [14]

- The Molecular Interaction Database (MINT, 2012 update, https://mint.bio.uniroma2.it/) [15]

Using the software Cytoscape (http://www.cytoscape.org) [16], a PPI network was visualized for Nor-NOHA and NOHA-associated genes.

Using the CytoNCA plug-in [17] (version 2.1.6, http://apps.cytoscape.org/apps/cytonca) in Cytoscape, degree centrality (DC), betweenness centrality (BC), and closeness centrality of the nodes of the PPI network were subjected to analysis to identify the hub proteins [18]. "Without weight." was set as the parameter.

\section{Module Analysis}

For module analysis of the PPI network, MCODE plug-in [19] (version 1.4.2; http://apps.cytoscape.org/apps/mcode; parameters set as degree cut-off $=2$, maximum depth $=100$, node score cut-off $=0.2$, and -core $=2$ ) in Cytoscape was used.

\section{Pathway Enrichment Analysis}

KEGG pathway enrichment analysis for the nodes of top modules was carried out with JEPETTO plug-in [20] in Cytoscape.

\section{Transcriptional Regulatory Network Construction}

Transcription factors (TFs) among nor-NOHA and NOHA-associated genes were searched and then their targets were identified using the transcriptional regulatory relationships unravelled by a sentence-based text-mining (TRRUST, http://www.grnpedia.org/trrust/) [21] database.

Finally, a transcriptional regulatory network of the hub proteins was constructed using Cytoscape [16]. 


\section{Results and Discussion}

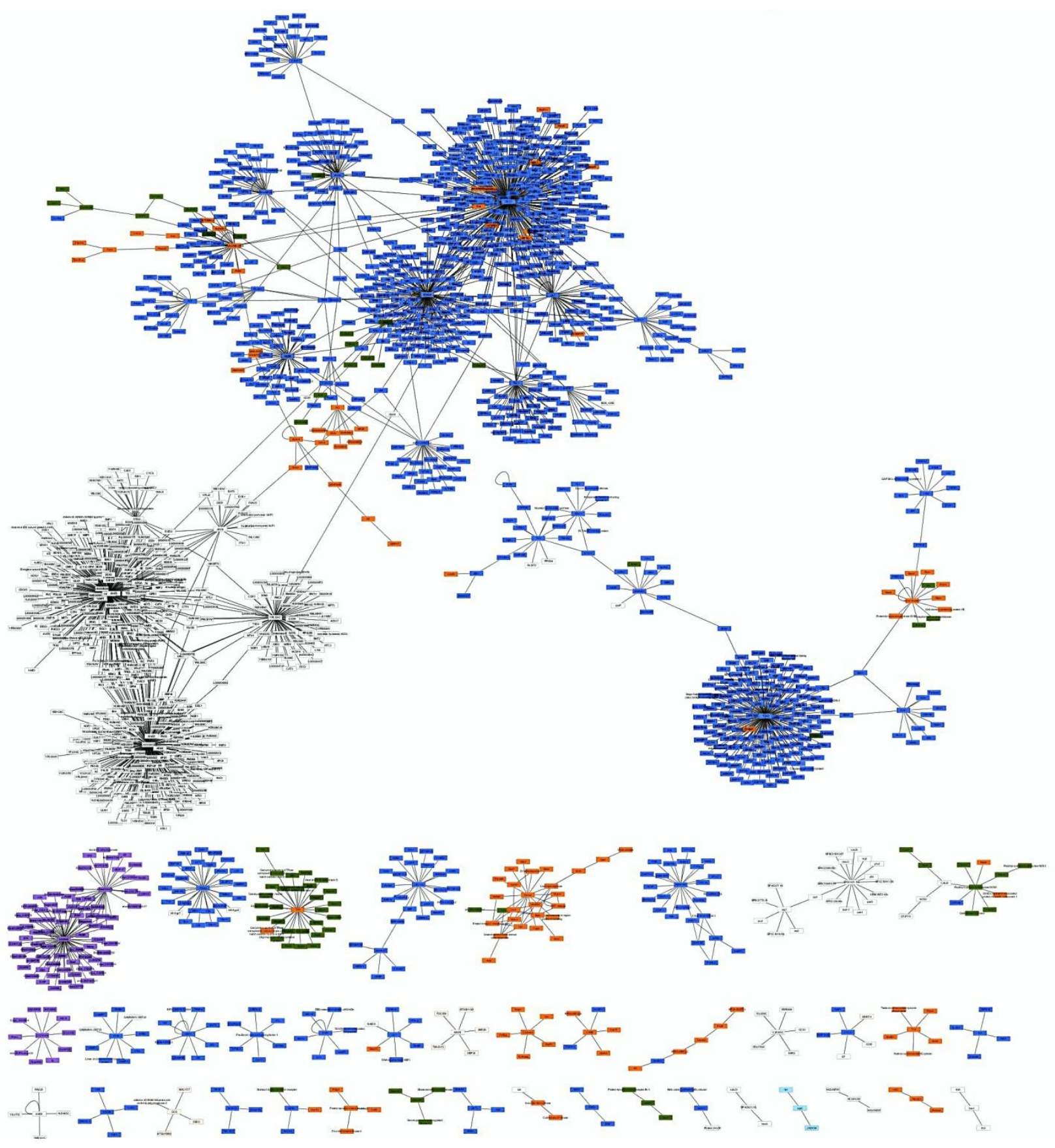

Figure 1: Protein-protein interaction network of nor-NOHA and NOHA associated genes. It contains 1996 nodes (proteins) and 2132 edges (interactions). 


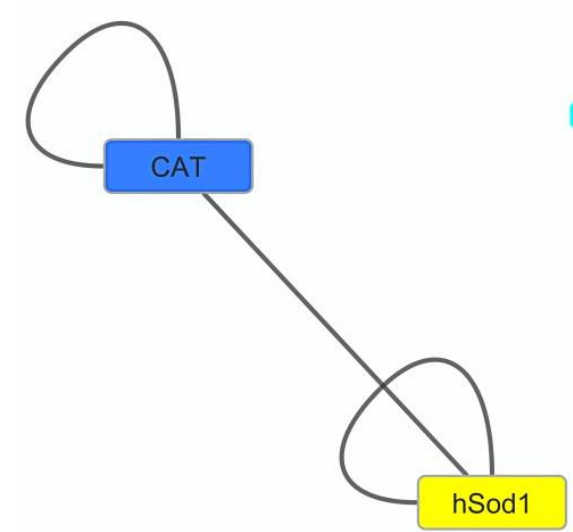

Module 1

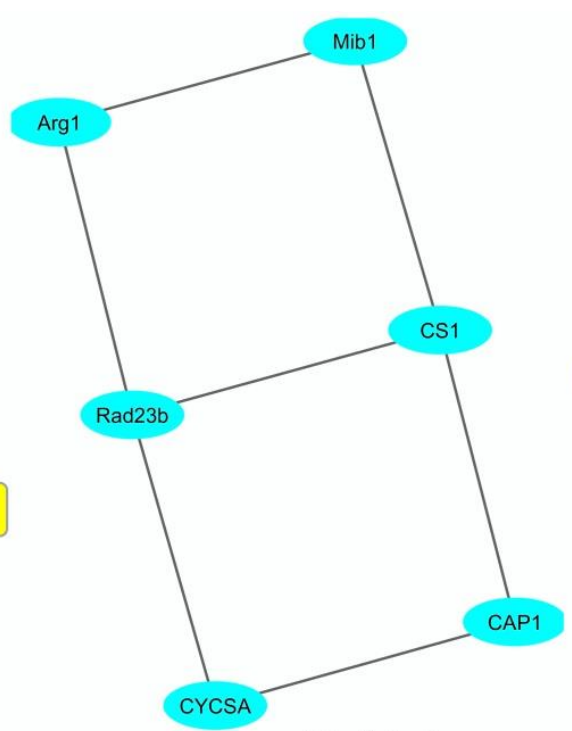

Module 2

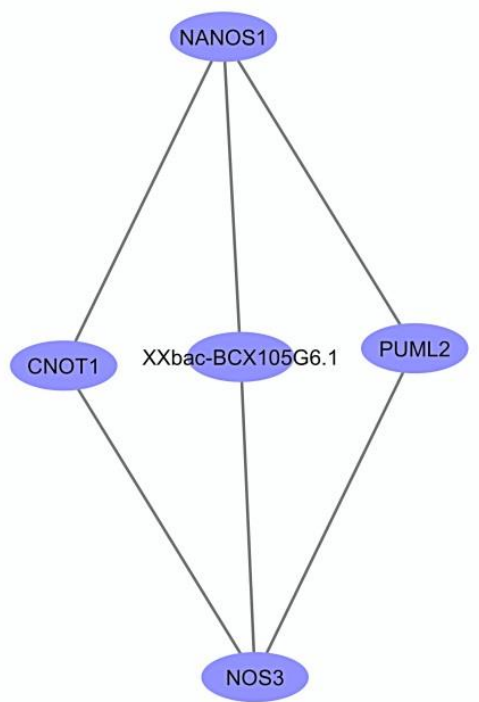

Module 3

Figure 2: Module 1, 2 and 3 derived using MCODE plug-in in Cytoscape with parameters set as degree cut-off $=2$, maximum depth $=100$, node score cut-off $=0.2$, and -core $=2$

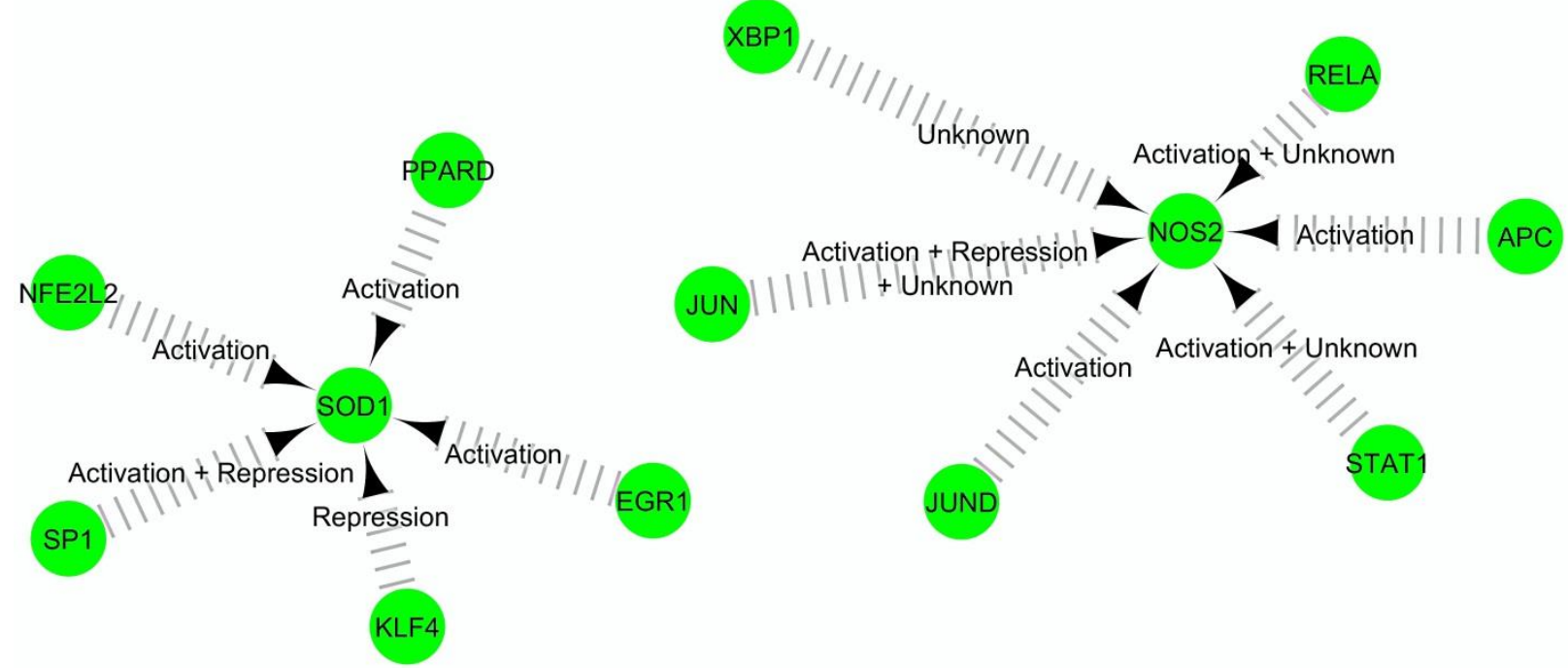

Figure 3: Transcriptional regulatory network of hub proteins SOD1 and NOS2 
Table 1: Nor-NOHA and NOHA associated genes

\begin{tabular}{|c|c|}
\hline Nor-NOHA associated genes & NOHA associated genes \\
\hline NOS3 & NOS1 \\
ARG2 & MARC2 \\
NOS2 & NOS2 \\
CD247 & MARC1 \\
NOS1 & NOS3 \\
SOD1 & ARG1 \\
DDAH1 & CYCS \\
IL6 & ODC1 \\
AGMAT & SOD1 \\
& AMD1 \\
& CAT \\
& ARG2 \\
& STX8 \\
& UROD \\
\hline
\end{tabular}

Table 2: Hub proteins in the PPI network according to Degree Centrality, Betweenness Centrality and Closeness Centrality using CytoNCA

\begin{tabular}{|c|c|c|c|c|c|}
\hline \multicolumn{2}{|l|}{} & \multicolumn{2}{r|}{} & \multicolumn{2}{r|}{ Closeness Centrality } \\
\hline Degree Centrality & \multicolumn{2}{|c|}{ Betweenness Centrality } & \multicolumn{2}{r|}{ Score } \\
\hline SODe & Score & Gene & Score & Gene & \\
\hline SOD1 & 236.0 & SOD & 1271250.132142436 & SOD & 0.0038243429647416014 \\
\hline AMD1 & 208.0 & CCS1 & 597881.8676198863 & SOD1 & 0.003820160825854615 \\
\hline NOS2 & 146.0 & AMD1 & 436818.27082915726 & GSH1 & 0.0038169885126128162 \\
\hline ODC1 & 68.0 & NOS2 & 308769.3082637827 & UBC & 0.003815663639055696 \\
\hline CuZnSOD & 62.0 & ODC1 & 195981.77444007777 & CAT & 0.0038166363585598754 \\
\hline
\end{tabular}




\begin{tabular}{|c|c|c|c|c|c|}
\hline CYCS & 49.0 & GSH1 & 142337.01101673584 & HSPABP2 & 0.003815154317167466 \\
\hline CAT & 49.0 & HUR & 132458.55204446075 & HSPH2 & 0.003815154317167466 \\
\hline CARB & 46.0 & CARB & 111604.5321192979 & KARP1 & 0.003814432239231872 \\
& & & & & \\
\hline NOS3 & 46.0 & NOS3 & 110840.35731586967 & NDPKB & 0.003814432239231872 \\
\hline
\end{tabular}

Table 3: KEGG pathway enrichment analysis result for the nodes of top modules

\begin{tabular}{|l|l|l|l|}
\hline Pathway or Process & XD-score & q-value & Overlap/Size \\
\hline Arginine and proline metabolism & 0.21151 & 0.09869 & $2 / 37$ \\
\hline Tryptophan metabolism & 0.14914 & 1.00000 & $1 / 26$ \\
\hline Nucleotide excision repair & 0.09053 & 1.00000 & $1 / 42$ \\
\hline Amyotrophic lateral sclerosis (ALS) & 0.08040 & 1.00000 & $1 / 47$ \\
\hline Peroxisome & 0.06672 & 1.00000 & $1 / 56$ \\
\hline VEGF signaling pathway & 0.05981 & 1.00000 & $1 / 62$ \\
\hline Amoebiasis & 0.03611 & 1.00000 & $1 / 98$ \\
\hline Protein processing in endoplasmic reticulum & 0.02407 & 1.00000 & $1 / 139$ \\
\hline Calcium signaling pathway & 0.02161 & 1.00000 & $1 / 152$ \\
\hline Metabolic pathways & 0.01404 & 1.00000 & $3 / 640$ \\
\hline
\end{tabular}

Table 4: Genes involved in the top 10 enriched KEGG pathways

\begin{tabular}{|l|l|}
\hline $\begin{array}{l}\text { Pathway or } \\
\text { Process }\end{array}$ & Genes Involved \\
\hline $\begin{array}{l}\text { Arginine and } \\
\text { proline } \\
\text { metabolism }\end{array}$ & $\begin{array}{l}\text { ABP1,DAO,PUM2,NANOS1,GOT2,GLUD1,CKM,CKB,NOS1,ARG1,SAT1, } \\
\text { SAT2,ARG2,ODC1,SRM,NOS2,RAD23B,CPS1,CAP1,OTC,ASS1,ALDH18A } \\
\text { 1,ASL,OAT,NOS3,SMS,P4HA2,ALDH2,GLS2,PYCR2,GLS,GLUL,MIB1,PY } \\
\text { CRL,AL,DH7A1,CKMT2,GAMT,GLUD2,unknown,GOT1,CAT,P4HA3,P4H } \\
\text { A1,EPRS,RARS,SIRT7 }\end{array}$ \\
\hline $\begin{array}{l}\text { Tryptophan } \\
\text { metabolism }\end{array}$ & $\begin{array}{l}\text { PUM2,NANOS1,ABP1,RAD23B,ECHS1,IL4I1,CAP1,NOS3,OGDH,ACAT2, } \\
\text { TPH1, } \\
\text { AANAT,ALDH2,ACAT1,WARS,ARG1,HADHA,IDO1,KYNU,MIB1,GCDH, } \\
\text { ALDH7A1,CYP1A1,EHHADH,WARS2,CAT,CYP1A2,HAAO,DDC,CYP1B1 }\end{array}$ \\
\hline
\end{tabular}




\begin{tabular}{|c|c|}
\hline & $\begin{array}{l}\text {,HADH,TDO2, } \\
\text { ACMSD }\end{array}$ \\
\hline $\begin{array}{l}\text { Nucleotide } \\
\text { excision repair }\end{array}$ & $\begin{array}{l}\text { ERCC5,GTF2H1,ERCC2,RPA3,RPA2,RPA1,ERCC3,ERCC4,CDK7,PCNA,E } \\
\text { RCC8, } \\
\text { DDB1,MNAT1,ERCC1,DDB2,RAD23A,XPC,GTF2H3,GTF2H2,RAD23B,XP } \\
\text { A, } \\
\text { CCNH,GTF2H5,POLD2,RFC1,RFC2,RFC5,POLE2,POLE,LIG1,POLD1,RPA } \\
\text { 4,RFC3,POLD3,POLD4,POLE3,POLE4,RFC4,PUM2,NANOS1,RBX1,CUL4 } \\
\text { A,CUL4B,CETN2,CAP1,NOS3,ARG1,MIB1,CAT }\end{array}$ \\
\hline $\begin{array}{l}\text { Amyotrophic } \\
\text { lateral } \\
\text { sclerosis } \\
\text { (ALS) }\end{array}$ & $\begin{array}{l}\text { BCL2L1,TP53,CYCS,BAD,BCL2,BID,CASP9,CASP1,BAX,APAF1,DAXX,P } \\
\text { PP3CA, } \\
\text { CASP3,SOD1,PPP3CB,MAPK14,MAPK11,RAC1,MAP3K5,PUM2,NANOS1, } \\
\text { MAP2K3,MAP2K6,MAPK12,MAPK13,GRIN2B,GRIN1,GRIN2A,CCS,NOS1 } \\
\text {,ARG1,GRIN2D,PRPH2,PRPH,NEFH,NEFM,ALS2,TNFRSF1A,TNFRSF1B, } \\
\text { RAD23B, } \\
\text { RAB5A,CAP1,GRIA1,unknown,NOS3,GRIA2,TOMM40,GRIN2C,PPP3CC, } \\
\text { MIB1,SLC1A2,CAT,DERL1 }\end{array}$ \\
\hline Peroxisome & $\begin{array}{l}\text { PHYH,PEX7,ABCD3,ACSL6,PUM2,NANOS1,GSTK1,FAR1,GNPAT,AGPS, } \\
\text { PEX19,PEX3,PEX10,PEX2,SLC25A17,PEX13,PXMP4,ABCD1,ABCD2,PEX } \\
\text { 14,PEX12,PEX16,PEX11B,PEX11A,ACAA1,PEX5,AGXT,HAO1,DDO,SCP2 } \\
\text { „EHHADH,ACOX1, } \\
\text { PEX1,PEX6,DAO,NOS2,RAD23B,PECR,CRAT,PMVK,SOD1,CAP1,IDH1,S } \\
\text { OD2, } \\
\text { NOS3,ACSL4,HSD17B4,ECH1,PRDX1,ARG1,ACOX3,MIB1,PRDX5,MVK, } \\
\text { PGAM5,HMGCL,ABCD4,EPHX2,CAT,PIPOX,HACL1,BAAT,IDH2 }\end{array}$ \\
\hline $\begin{array}{l}\text { VEGF } \\
\text { signaling } \\
\text { pathway }\end{array}$ & $\begin{array}{l}\text { PTK2,SRC,PLCG1,PXN,PIK3R1,RAF1,PRKCA,AKT1,KDR,HRAS,PLCG2,S } \\
\text { H2D2A, } \\
\text { BAD,MAPK3,MAPK1,MAP2K1,KRAS,MAP2K2,RAC1,CDC42,NFATC1,P } \\
\text { RKCG, } \\
\text { VEGFA,PPP3CA,PPP3CB,CASP9,MAPKAPK2,NOS3,AKT2,PUM2,NANOS } \\
\text { 1,NFATC4,MAPK14,NFATC2,HSPB1,RAC2,PIK3CA,PIK3CD,PIK3CB,PLA } \\
\text { 2G4A,MAPKAPK3,MAPK13,PIK3R3,PIK3R2,SHC2,PIK3CG,PLA2G2A,PL } \\
\text { A2G1B,MAPK11,PLA2G5,PIK3R5,PRKCB,RAD23B,MAPK12,CAP1,NFAT } \\
\text { 5,NFATC3,unknown,AKT3,PLA2G10,PTGS2,ARG1,PPP3CC,MIB1,RAC3,C } \\
\text { AT,SPHK1,JMJD7-PLA2G4B, } \\
\text { TBC1D3F,PLEKHG2,OPHN1,CDC42BPG,CDC42SE1,TCL1A,PDE3B,TCL1 } \\
\text { B,ICMT,PLCE1,SHOC2,RASSF2,NRP1,NRAS,KSR1,ALS2CR12,EPX,FOXP } \\
\text { 3,DUSP9,DUSP10,MAPKSP1,DUSP5,PTPRR,PTPN5,NRGN,DUSP2,ZNHIT } \\
\text { 1,NOXA1,C11orf17,PLA2R1,MIR1538,SPHK2 }\end{array}$ \\
\hline Amoebiasis & $\begin{array}{l}\text { PTK2,ACTN1,VCL,PIK3R1,ITGB2,TGFB1,COL2A1,TGFB2,LAMB1,LAMA } \\
\text { 5,LAMC1,PRKCA,GNA15,PLCB1,ACTN4,LAMA3,NOS2,RELA,PRKACA, } \\
\text { NFKB1,RAB7A,RAB5A,ITGAM,CD14,PRKACB,PRKACG,PRKCB,PUM2, } \\
\text { NANOS1,LAMB3,FN1,COL1A2,COL4A4,COL1A1,COL4A2,COL4A1,COL } \\
\text { 4A6,COL5A3,GNAQ,PIK3CA,PLCB2,PIK3CD,PIK3CB,IL1R1,TLR2,PIK3R } \\
\text { 3,TLR4,PIK3R2,COL5A1,C8B,C8A,C9,C8G,RAB5C,LAMB2,LAMC2,TGFB } \\
\text { 3,PIK3CG,PIK3R5,IL1B,ARG1,ARG2,IL12B,IL12A,IL1R2,CTSG,SERPINB1 }\end{array}$ \\
\hline
\end{tabular}




\begin{tabular}{|c|c|}
\hline & $\begin{array}{l}\text { 3,ACTN2,PRKCG,CASP3,GNA11,SERPINB2,RAD23B, } \\
\text { COL3A1,CD1D,GNAS,SERPINB1,IFNG,CAP1,CSF2,SERPINB3,PLCB3,CO } \\
\text { L11A1, } \\
\text { NOS3,RAB5B,SERPINB9,ADCY1,LAMA1,IL6,HSPB1,PLCB4,CXCL1,IL8, } \\
\text { LAMA4,MIB1,SERPINB4,PRKX,IL10,SERPINB6,MUC2,CAT,COL11A2,G } \\
\text { NA14,LAMA2, } \\
\text { COL5A2 }\end{array}$ \\
\hline $\begin{array}{l}\text { Protein } \\
\text { processing in } \\
\text { endoplasmic } \\
\text { reticulum }\end{array}$ & $\begin{array}{l}\text { UBQLN1,UBQLN4,P4HB,SSR4,SSR3,SEC63,SEC62,HSPA5,SEC61B,VCP, } \\
\text { ATXN3,HSPA8,HSP90B1,UFD1L,NPLOC4,NSFL1C,PLAA,UBE4B,AMFR, } \\
\text { DERL1, } \\
\text { RAD23A,RAD23B, } \\
\text { TRAF2,MAP3K5,ERN1,MAPK9,HSPA1A,HSPBP1,BCL2, } \\
\text { DNAJA2,BAG2,PPP1R15A,EIF2AK2,DNAJC3,STUB1,DNAJB11,DNAJB1, } \\
\text { HSPA2,UBQLN2,RPN1,SYVN1,ERP29,DNAJA1,CANX,PRKCSH,BCAP31, } \\
\text { PDIA3,EDEM1,MBTPS1,MAPK8,MAP2K7,BAK1,BAX,PUM2,NANOS1,HS } \\
\text { P90AB1,SKP1, } \\
\text { HSPH1,SAR1B,EIF2AK3,NFE2L2,ATF4,RBX1,CUL1,FBXO2,CALR,HSP90 } \\
\text { AA1,NOS3,EIF2AK1,DNAJC10,SEC23B,SIL1,DNAJC1,SEC24B,SEC13,ST } \\
\text { T3A,RPN2,DAD1,TUSC3,STT3B,UBE2D3,RNF5,NGLY1,UBE2E1,UBE2G1 } \\
\text {,SEC24C,SEC23A, } \\
\text { ERO1LB,ERO1L,TRAM1,GANAB,EIF2S1,SEC24D,SEC31A,CRYAB,CRY } \\
\text { AA,PARK2,UBE2D2,UBE2D1,DDIT3,UBE2E3,UBE2G2,UBE2J2,HERPUD1 } \\
\text {,SAR1A,DNAJC5,MBTPS2,HYOU1,CAPN1,UBE2E2,CAP1,SEC61A1,MAP } \\
\text { K10,SEC61A2,ATF6, } \\
\text { RRBP1,ERLEC1,UBE2D4,SEL1L,SEC24A,DERL2,LMAN1,CAPN2,ARG1,P } \\
\text { DIA4,EDEM2,MOGS,XBP1,DERL3,UGGT1,CKAP4,OS9,MIB1,UGGT2,SS } \\
\text { R1,EIF2AK4,PREB,MAN1A2,WFS1,MAN1C1,CAT,DNAJB2,HSPA6,PDIA6 } \\
\text {,SSR2,HSPA1L }\end{array}$ \\
\hline $\begin{array}{l}\text { Calcium } \\
\text { signaling } \\
\text { pathway }\end{array}$ & $\begin{array}{l}\text { PLCG1,EGFR,ERBB2,PLCG2,PDGFRB,GRIN2A,PDGFRA,AGTR1,PTGFR, } \\
\text { PTGER1,PRKCA,GRM5,NOS1,ITPKA,GNA15,ATP2B2,MYLK,ATP2B1,IT } \\
\text { PKB,ADRA1B, } \\
\text { PLCB1,AVPR1A,GRM1,PRKCG,GRIN1,GRIN2D,PRKACA,PRKACB,PRK } \\
\text { ACG,ITPR1,RYR1,PHKA1,ITPR2,CACNA1C,PLN,RYR2,PPP3CA,ATP2B4, } \\
\text { PPP3CB,CAMK4,CALM1,PLCD1,CAMK2G,PHKA2,CAMK2A,PHKB,PHK } \\
\text { G2,PLCB3,PHKG1,ADCY8,PDE1A,PRKCB,PUM2,SLC25A5,NANOS1,SLC } \\
\text { 25A6,SLC25A4,ADRB1,PTK2B,ERBB4,ERBB3,TBXA2R,GNA11,GNAQ,H } \\
\text { TR2A,ITPR3,ADRB2,CHRM2,TRPC1,EDNRB,EDNRA,HTR2B,BDKRB2,P } \\
\text { LCB2,HTR6,CHRM3,BDKRB1,HTR2C,ADCY3,CAMK2B,PPID,F2R,GNAS, } \\
\text { ADCY2,LHCGR,ADRB3,CAMK2D,ATP2A2,PTGER3,CACNA1S,ARG1,GN } \\
\text { A14,NOS3,DRD1,ATP2A1,RYR3,ADCY1,ADRA1A,SLC8A3,SLC8A2,SLC8 } \\
\text { A1,ADRA1D,P2RX2,P2RX1,P2RX3,CCKAR,CCKBR,CACNA1G,CD38,TA } \\
\text { CR2,TACR1,TACR3,NOS2,PLCE1,RAD23B,VDAC3,VDAC2,CAP1,unknow } \\
\text { n, } \\
\text { ADORA2B,CACNA1D,CACNA1A,ATP2A3,P2RX7,CACNA1B,GRIN2C,P2 } \\
\text { RX5,PLCD4,CACNA1E,P2RX6,PPP3CC,MYLK2,PLCB4,NTSR1,OXTR,AV } \\
\text { PR1B,TNNC2, }\end{array}$ \\
\hline
\end{tabular}




\begin{tabular}{|c|c|}
\hline & $\begin{array}{l}\text { MIB1,TNNC1,PTAFR,CALML5,BST1,DRD5,PRKX,HRH2,CHRM1,ADCY7 } \\
\text {,CAT,GRPR,SPHK1,CALML3,P2RX4,PLCD3,TRHR,CHRNA7,HTR7,CACN } \\
\text { A1H,CHRM5,CALM2,CAMKK2,BCAR3,DAB1,TNS3,CACNB3,RNF41,NR } \\
\text { G2,HTR4,BANK1,VDAC1P1,PPIF,CABP2,RIC8B,TAC4,CAMK2N2,AVP,S } \\
\text { LN,ADORA2A,CHRFAM7A,CACNA1F,SPHK2,CACNA1I }\end{array}$ \\
\hline & 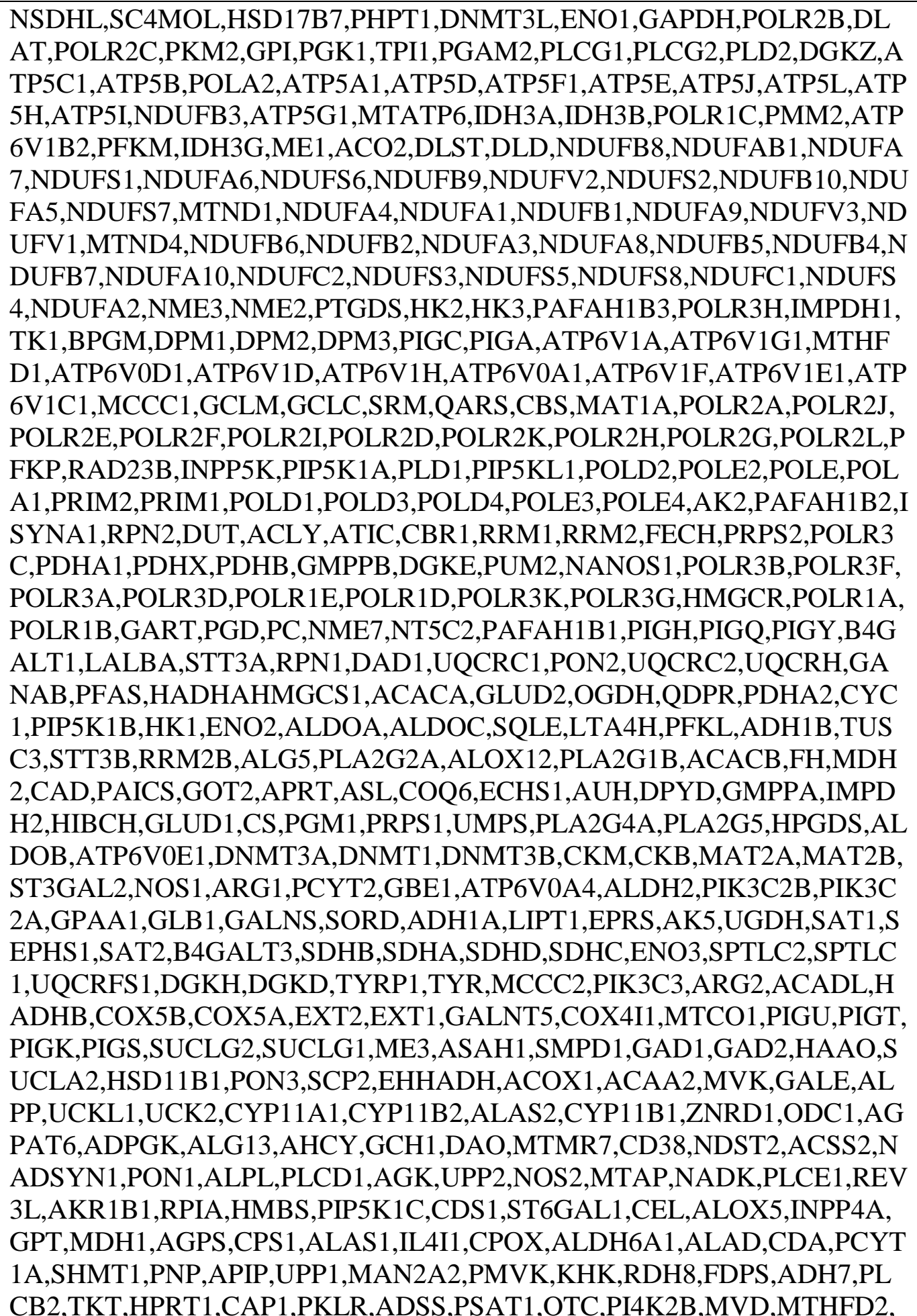 \\
\hline
\end{tabular}




\begin{tabular}{|l|l|}
\hline TRIT1,LCT,IDH1,CTPS2,UQCRQ,ASS1,HEXB,PPAP2B,INPPL1,IMPA2,AC \\
ADVL,ALG10,ALG10B,AK1,ITPKA,PLCB3,ALDH18A1,ACAA1,BCAT1,A \\
GXT,OAT,DGKA,NOS3,OCRL,PPAP2A,DGKQ,HYAL2,COASY,SMS,ACS \\
L4,INPP5A,ATP6V0B,ACAT2,LDHA,PLA2G10,PIGB,ASNS,COMT,GLYC \\
TK,ATP6AP1,SI,TPH1,AANAT,ITPKB,GUSB,TH,FBP1,DCTD,HSD17B10,P \\
4HA2,LIAS,PANK2,MGLL,ACAT1,ATP6V0C,HSD17B4,NT5E,GDA,GLS2, \\
CRLS1,SYNJ1,SYNJ2,PYCR2,HSD3B2,FDFT1,NDUFA4L2,BCKDHB,GLS, \\
SLC27A5,PHGDH,LIPC,GCK,PLCD4,FDXACB1,ACOX3,MTMR2,PGS1,SH \\
MT2,MTR,GLUL,ATP6V1B1,MOGS,TRDMT1,IDO1,PLCB1,PTGS1,NANS, \\
KYNU,FLAD1,PSPH,CTPS,GGT1,GAL3ST1,PPAT,PMM1,ITPK1,PLCB4,M \\
ECR,COX6B1,COX6C,ADK,MPI,HAO1,MIB1,NAT2,NAT1,UGT8,PCYT1B, \\
COX17,PPCDC,QPRT,DBT,NNT,COQ7,IMPA1,PYCRL,MTMR6,ADI,INP \\
P1,GCDH,SMPD2,BST1,POLG,PI4KB,ACSL6,ALDH7A1,PAH,MAN1A2,C \\
KMT2,PAPSS1,PPAP2C,ST3GAL4,MPST,GAMT,CHKA,LDHB,PDXK,GM \\
PS,CMAS,CBR3,PGLS,AASDHPPT,NME5,Unknown,MTCO2,GNPDA1,AD \\
A,GOT1,GALNT6,SPR,UXS1,TPO,DHCR24,AMPD2,PRDX6,AMPD1,CTH, \\
ACADM,HMGCL,AKR1A1,MAN1C1,GALNT12,GALNT14,GALNT10,ST3 \\
GAL3,ALG2,HYI,CHPF,MGAT1,EPHX2,CAT,PIPOX,PTGES2,MUT,UGP2, \\
ST6GALNAC6,SGMS1,PTGES,G6PD,HYAL3,SPHK1,IDUA,CYP2E1,CYP2 \\
C9,CYP1A2,CYP17A1,CYP2C19,MTHFR,TALDO1,JMJD7PLA2G4B,CDO1 \\
,FBP2,COX4I2,GGT7,GALNTL2,AOC3,G6PC,PPOX,DDC,CNDP1,UGCG,D \\
TYMK,PAPSS2,B3GNT4,MTHFS,PLCD3,SMPD4,BAAT,PGAP1,GRHPR,C \\
YP19A1,TYMS,PTS,HADH,ETNK2,GALNT1,TKTL2,BPNT1,GALNT2,DE \\
GS1,GALNT13,PGAM1,GALNT11,MTCO3,PLA2G7,UQCRB,DPYS,MGAT \\
4B,INPP5E,P4HA3,DHCR7,GALK2,AHCYL1,TDO2,ACMSD,GFPT1,ATP5 \\
G3,ALPI,PANK4,AHCYL2,FUT4,ALOX12B,UROS,CMPK1,SGPL1,GCNT1, \\
ANPEP,FASN,DCXR,ADH5,ENPP1,RPE,AKR1B10,GLDC,GBA,IDH2,NT5 \\
C1B,MT-ND2,INPP5B,MT-ND3,ACAD8,P4HA1
\end{tabular}

Table 5: Transcription factors targeting nor-NOHA and NOHA associated genes

\begin{tabular}{|c|c|c|c|c|}
\hline$\#$ & Key TF & Description & Target genes & Mode of action \\
\hline \multirow{7}{*}{1} & \multirow{7}{*}{ SP1 } & \multirow{7}{*}{ Sp1 transcription factor } & CAT & Unknown \\
\hline & & & IL6 & Activation \\
\hline & & & NOS1 & Unknown \\
\hline & & & NOS3 & Activation \\
\hline & & & NOS3 & Unknown \\
\hline & & & ODC1 & Unknown \\
\hline & & & SOD1 & Activation \\
\hline
\end{tabular}




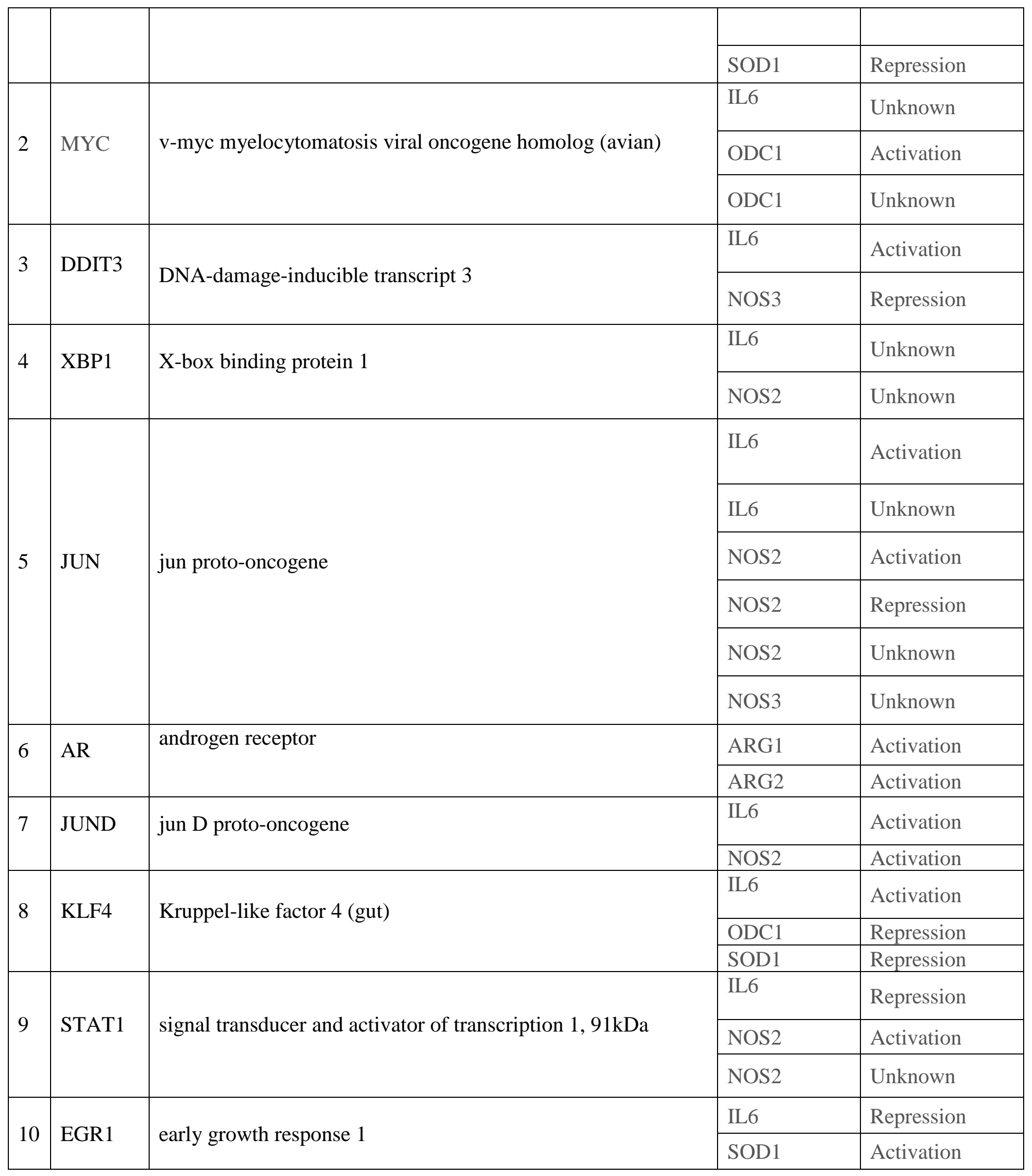




\begin{tabular}{|c|c|c|c|c|}
\hline \multirow{2}{*}{11} & \multirow{2}{*}{ PPARD } & \multirow{2}{*}{ peroxisome proliferator-activated receptor delta } & CAT & Activation \\
\hline & & & SOD1 & Activation \\
\hline \multirow{2}{*}{12} & \multirow{2}{*}{ APC } & \multirow{2}{*}{ adenomatous polyposis coli } & NOS2 & Activation \\
\hline & & & ODC1 & Repression \\
\hline \multirow{2}{*}{13} & \multirow{2}{*}{ NFE2L2 } & \multirow{2}{*}{ nuclear factor (erythroid-derived 2)-like 2} & CAT & Activation \\
\hline & & & SOD1 & Activation \\
\hline \multirow[t]{2}{*}{14} & \multirow[t]{2}{*}{ XBP1 } & \multirow[t]{2}{*}{ X-box binding protein 1} & IL6 & Unknown \\
\hline & & & NOS2 & Unknown \\
\hline \multirow{7}{*}{15} & \multirow{7}{*}{ RELA } & \multirow{7}{*}{ v-rel reticuloendotheliosis viral oncogene homolog A (avian) } & IL6 & Activation \\
\hline & & & IL6 & Repression \\
\hline & & & IL6 & Unknown \\
\hline & & & NOS1 & Activation \\
\hline & & & NOS2 & Activation \\
\hline & & & NOS2 & Unknown \\
\hline & & & NOS3 & Activation \\
\hline \multirow{2}{*}{16} & \multirow{2}{*}{ CREB1 } & \multirow[t]{2}{*}{ cAMP responsive element binding protein 1} & IL6 & Activation \\
\hline & & & ODC1 & Unknown \\
\hline
\end{tabular}

In this study, a total of 19 nor-NOHA and NOHA-associated genes were identified from GeneCards, STICH and CTD databases (Table 1) and their protein-protein interaction network was constructed (Figure 1). Based on Betweenness Centrality, Closeness Centrality, and Degree Centrality scores SOD, SOD1, AMD1, and NOS2 were established as hub nodes in the ProteinProtein Interaction network of these genes and their interactions (Table 2). Three distinct modules (modules 1, 2 and 3) of the PPI network were identified (Figure 2). Arginine and proline metabolism, tryptophan metabolism, nucleotide excision repair, amyotrophic lateral sclerosis (ALS) and peroxisome were the top 5 pathways/processes the proteins in these modules were found to be significantly involved in (Table 3). Only 2 out of the 4 hub proteins, namely SOD1 and NOS2 were present in the TRRUST database of transcription factors (Table 5). So a transcriptional regulatory network of them was constructed (Figure 3). SOD1 has been found to be the target of transcription factors SP1, KLF4, EGR1, PPARD and NFE2L2 while NOS2 was found to be the target of transcription factors XBP1, JUN, JUND, STAT1, APC, XBP1, and RELA. 
Superoxide dismutase has long been considered a target for the selective killing of cancer cells [22]. It has also been labeled as body's natural cancer fighter [23]. SOD1 however has been only recently been proposed as a novel target for cancer therapy [24] and its role in cancer is also being revealed [25]. More recently being elucidated is the role of AMD1 in cancer which has been found to be upregulated in human prostate cancer [26]. NOS2 is another one which has been recently being seen as a emerging target for cancer treatment [27][28].

\section{Conclusion}

To conclude, a total of 19 nor-NOHA and NOHA-associated genes have been identified. SOD, SOD1, AMD1, and NOS2 have been revealed as key players in nor-NOHA and NOHA mediated pathways. Interestingly, these proteins have been shown to be associated with cancer in other studies as well. Therefore, this study has further validated the arguments for targeting them for treating cancer.

\section{References}

[1] J. B. Ochoa, J. Strange, P. Kearney, G. Gellin, E. Endean, and E. Fitzpatrick, "Effects of L-Arginine on the Proliferation of T Lymphocyte Subpopulations," J. Parenter. Enter. Nutr., vol. 25, no. 1, pp. 23-29, Jan. 2001.

[2] G. Wu and S. M. Morris, "Arginine metabolism: nitric oxide and beyond.," Biochem. J., vol. 336 ( Pt 1), pp. 1-17, Nov. 1998.

[3] K. Miyanaka et al., "Immunohistochemical localization of arginase II and other enzymes of arginine metabolism in rat kidney and liver.," Histochem. J., vol. 30, no. 10, pp. 74151, Oct. 1998.

[4] M. Munder et al., "Arginase I is constitutively expressed in human granulocytes and participates in fungicidal activity," Blood, vol. 105, no. 6, pp. 2549-2556, Mar. 2005.

[5] T. Gotoh, M. Araki, and M. Mori, "Chromosomal Localization of the Human Arginase II Gene and Tissue Distribution of Its mRNA," Biochem. Biophys. Res. Commun., vol. 233, no. 2, pp. 487-491, Apr. 1997.

[6] O. Braissant, T. Gotoh, M. Loup, M. Mori, and C. Bachmann, "L-arginine uptake, the citrulline-NO cycle and arginase II in the rat brain: an in situ hybridization study.," Brain Res. Mol. Brain Res., vol. 70, no. 2, pp. 231-41, Jul. 1999.

[7] R. Singh, S. Pervin, A. Karimi, S. Cederbaum, and G. Chaudhuri, "Arginase activity in human breast cancer cell lines: N(omega)-hydroxy-L-arginine selectively inhibits cell proliferation and induces apoptosis in MDA-MB-468 cells.," Cancer Res., vol. 60, no. 12, pp. 3305-12, Jun. 2000.

[8] S. M. Mumenthaler et al., "Expression of arginase II in prostate cancer.," Int. J. Oncol., 
vol. 32, no. 2, pp. 357-65, Feb. 2008.

[9] F. Wu and H. Gehring, "Structural requirements for novel coenzyme-substrate derivatives to inhibit intracellular ornithine decarboxylase and cell proliferation," FASEB J., vol. 23, no. 2, pp. 565-574, Feb. 2009.

[10] P. C. Rodriguez et al., "Arginase I Production in the Tumor Microenvironment by Mature Myeloid Cells Inhibits T-Cell Receptor Expression and Antigen-Specific T-Cell

Responses," Cancer Res., vol. 64, no. 16, pp. 5839-5849, Aug. 2004.

[11] M. Safran et al., "GeneCards Version 3: the human gene integrator," Database, vol. 2010, no. 0, p. baq020-baq020, Aug. 2010.

[12] D. Szklarczyk, A. Santos, C. von Mering, L. J. Jensen, P. Bork, and M. Kuhn, "STITCH 5: augmenting protein-chemical interaction networks with tissue and affinity data," Nucleic Acids Res., vol. 44, no. D1, pp. D380-D384, Jan. 2016.

[13] A. P. Davis et al., "The Comparative Toxicogenomics Database: update 2017," Nucleic Acids Res., vol. 45, no. D1, pp. D972-D978, Jan. 2017.

[14] A. Chatr-aryamontri et al., "The BioGRID interaction database: 2015 update," Nucleic Acids Res., vol. 43, no. D1, pp. D470-D478, Jan. 2015.

[15] L. Licata et al., "MINT, the molecular interaction database: 2012 update," Nucleic Acids Res., vol. 40, no. D1, pp. D857-D861, Jan. 2012.

[16] R. Saito et al., “A travel guide to Cytoscape plugins," Nat. Methods, vol. 9, no. 11, pp. 1069-1076, Nov. 2012.

[17] M. Li, Y. Lu, Z. Niu, and F.-X. Wu, "United Complex Centrality for Identification of Essential Proteins from PPI Networks," IEEE/ACM Trans. Comput. Biol. Bioinforma., vol. 14, no. 2, pp. 370-380, Mar. 2017.

[18] X. He and J. Zhang, "Why Do Hubs Tend to Be Essential in Protein Networks?"

[19] G. D. Bader and C. W. Hogue, "An automated method for finding molecular complexes in large protein interaction networks," BMC Bioinformatics, vol. 4, no. 1, p. 2, Jan. 2003.

[20] C. Winterhalter, P. Widera, and N. Krasnogor, "JEPETTO: a Cytoscape plugin for gene set enrichment and topological analysis based on interaction networks," Bioinformatics, vol. 30, no. 7, pp. 1029-1030, Apr. 2014.

[21] H. Han et al., "TRRUST: a reference database of human transcriptional regulatory interactions," Sci. Rep., vol. 5, no. 1, p. 11432, Sep. 2015.

[22] P. Huang, L. Feng, E. A. Oldham, M. J. Keating, and W. Plunkett, "Superoxide dismutase as a target for the selective killing of cancer cells.," Nature, vol. 407, no. 6802, pp. 390395, Sep. 2000.

[23] T. M. Brasky et al., "Serum Phospholipid Fatty Acids and Prostate Cancer Risk: Results From the Prostate Cancer Prevention Trial," Am. J. Epidemiol., vol. 173, no. 12, pp. 14291439, Jun. 2011. 
[24] L. Papa, G. Manfredi, and D. Germain, "SOD1, an unexpected novel target for cancer therapy.," Genes Cancer, vol. 5, no. 1-2, p. 15, Apr. 2014.

[25] S. Zhang et al., "The superoxide dismutase 1 3'UTR maintains high expression of the SOD1 gene in cancer cells: The involvement of the RNA-binding protein AUF-1," Free Radic. Biol. Med., vol. 85, pp. 33-44, Aug. 2015.

[26] A. Zabala-Letona et al., "mTORC1-dependent AMD1 regulation sustains polyamine metabolism in prostate cancer," Nature, vol. 547, no. 7661, pp. 109-113, Jun. 2017.

[27] D. D. Thomas and D. A. Wink, "NOS2 as an Emergent Player in Progression of Cancer," Antioxid. Redox Signal., vol. 26, no. 17, pp. 963-965, Jun. 2017.

[28] D. Basudhar et al., "Coexpression of NOS2 and COX2 accelerates tumor growth and reduces survival in estrogen receptor-negative breast cancer.," Proc. Natl. Acad. Sci. U. S. A., vol. 114, no. 49, pp. 13030-13035, Dec. 2017. 